\title{
Open Access Open Access Scientific Reports

\section{Azacitidine Causes Prolonged Neutropenia in an Elderly Patient with Primary Myelofibrosis Transformed To Acute Myeloid Leukemia}

\section{Richard Cheung Yiu* and Grace Li Shan Kam}

Department of Hematology, Singapore General Hospital, Singapore

\begin{abstract}
Leukemic transformation of primary myelofibrosis confers a dismal prognosis despite aggressive treatment with intensive chemotherapy and stem cell transplantation. Elderly patients with myelofibrosis transformed to acute leukemia have limited treatment options and are often excluded from intensive chemotherapy or stem cell transplantation. The hypomethylating agent, azacitidine, is a potential treatment for this group of patients. Azacitidine is relatively well tolerated by elderly patients with myelodysplastic syndromes, but in patients with background myelofibrosis, there is a concern about its hematologic toxicities because the hematologic recovery was found to be slower in patients with extensive marrow fibrosis after chemoradiotherapy and stem cell transplantation. To our knowledge, limited data is available on the hematologic adverse effects in this particular group of patients with underlying myelofibrosis. Here we report a case of leukemic transformation of myelofibrosis in an elderly patient who was treated with azacitidine for the leukemic transformation. He developed severe persistent neutropenia after the second cycle of azacitidine, precluding further cycles of azacitidine. Delayed recovery of blood counts in patients with background marrow fibrosis after azacitidine has not been discussed in previous studies and this raises questions on the association between significant marrow fibrosis and hematologic recovery after use of hypomethylating agents.
\end{abstract}

Keywords: Myelofibrosis; Leukemic Transformation; Azacitidine

\section{Introduction}

Approximately 5 to $15 \%$ of patients with Myelofibrosis (MF) will undergo transformation to Acute Myeloid Leukemia (AML) over a 10 -year period [1,2]. Leukemic transformation of primary MF confers a dismal prognosis despite treatment with intensive chemotherapy and allogeneic Stem Cell Transplantation (SCT). In particular, elderly patients with leukemic transformation have very poor clinical outcomes and may be excluded from intensive chemotherapy or stem cell therapy due to poor performance status and/or co-morbidities [3]. A recent study suggested a potential role of azacitidine, a DNA methyltransferase inhibitor or hypomethylating agent, as a treatment for patients with Philadelphia-negative Myeloproliferative Neoplasms (MPN) transformed to Myelodysplastic Syndrome (MDS) or AML [4]. Azacitidine is relatively well tolerated by elderly patients with MDS [5, 6], but in patients with background MF, there is a concern about its hematologic toxicities because the hematologic recovery was found to be slower in patients with extensive marrow fibrosis after chemoradiotherapy and SCT [7]. Here we report a case of primary MF transformed to AML with prolonged neutropenia after treatment with azacitidine.

\section{Case report}

A 73-years old gentleman with background history of hypertension was diagnosed to have primary MF in July 2006. At the time of diagnosis, he had splenomegaly of $10 \mathrm{~cm}$ below the left costal margin; his hemoglobin and platelet counts were normal but white blood cell count was elevated $\left(16.7 \times 10^{9} / \mathrm{L}\right)$ and blood film showed evidence of leucoerythroblastic picture. His MF disease was stable for almost 4 years. Then he developed progressive thrombocytopenia and increasing peripheral blasts in blood film since February 2010. His peripheral myeloid blasts increased to $45 \%$ with a development of myeloid sarcoma at his chest wall confirmed by histology in November 2010. At that point of time, his hemoglobin level was $10.9 \mathrm{~g} / \mathrm{dL}$, white cell count was $20.6 \times 10^{9} / \mathrm{L}$, neutrophil count was $4.73 \times 10^{9} / \mathrm{L}$, platelet count was
$33 \times 10^{9} / \mathrm{L}$ and blasts were $45 \%$ in peripheral blood. He was confirmed to have leukemic transformation of his MF. Due to his age and poor performance status, he was treated with azacitidine $75 \mathrm{mg} / \mathrm{m}^{2}$ daily for 7 days starting from 9 December 2010. At the start of treatment, his hemoglobin level was $10.9 \mathrm{~g} / \mathrm{dL}$, white cell count was $12.0 \mathrm{x} 10^{9} / \mathrm{L}$, platelet count was $20 \times 10^{9} / \mathrm{L}$ and $60 \%$ blasts were present in peripheral blood. A second cycle of azacitidine of the same dosage and schedule was given from 6 January 2011. After the second cycle of azacitidine, his absolute neutrophil count dropped from normal to $0.32 \times 10^{9} / \mathrm{L}$ with progressive worsening of the neutropenia. His peripheral blood blasts disappeared from 10 February 2011 but his neutropenia persisted. Therefore, further cycle of azacitidine was deemed not suitable. Till 9 March 2011 before his demise, his white cell count was $0.65 \times 10^{9} / \mathrm{L}$ with only few neutrophils detectable in the blood film. His white blood cell, neutrophil and blast counts from the start of azacitidine treatment to his death were illustrated in Figure 1.

In summary, this patient received 2 cycles of azacitidine therapy for leukemic transformation of myelofibrosis. He developed worsening neutropenia after the second cycle of treatment with no evidence of myeloid recovery at 9 weeks after the second cycle of azacitidine.

\section{Discussion}

An earlier study reported a slower rate of myeloid recovery and longer duration of platelet and red blood cell transfusion dependency in patients with severe marrow fibrosis after allogeneic bone marrow

*Corresponding author: Richard Cheung Yiu, Department of Hematology, Singapore General Hospital, Singapore, Tel: 656326 6604; Fax: 6562250210 Email: richard.yiu@sgh.com.sg

Received November 12, 2011; Published July 27, 2012

Citation: Yiu RC, Shan Kam GL (2012) Azacitidine Causes Prolonged Neutrope nia in an Elderly Patient with Primary Myelofibrosis Transformed To Acute Myeloid Leukemia. 1: 200. doi:10.4172/scientificreports.200

Copyright: (c) 2012 Yiu RC, et al. This is an open-access article distributed unde the terms of the Creative Commons Attribution License, which permits unrestricted use, distribution, and reproduction in any medium, provided the original author and source are credited. 
Citation: Yiu RC, Shan Kam GL (2012) Azacitidine Causes Prolonged Neutropenia in an Elderly Patient with Primary Myelofibrosis Transformed To Acute Myeloid Leukemia. 1: 200. doi:10.4172/scientificreports.200

1A

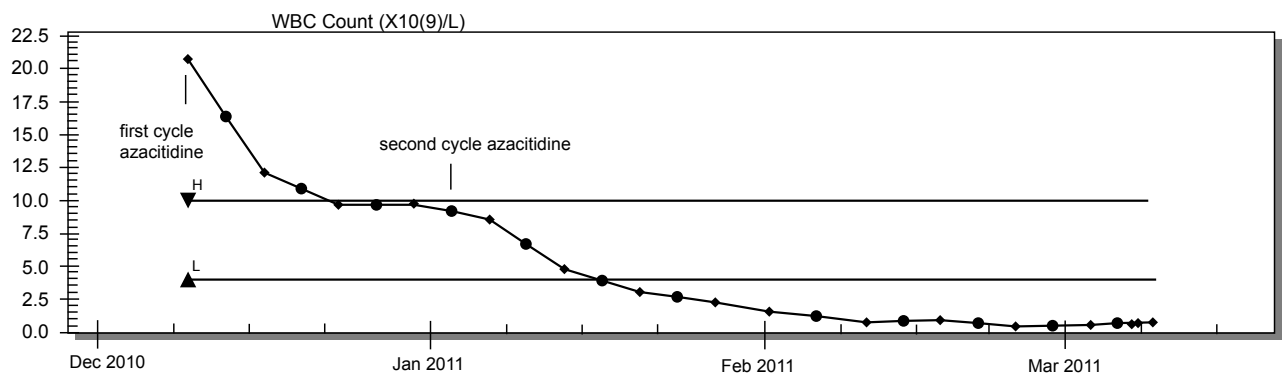

$1 B$

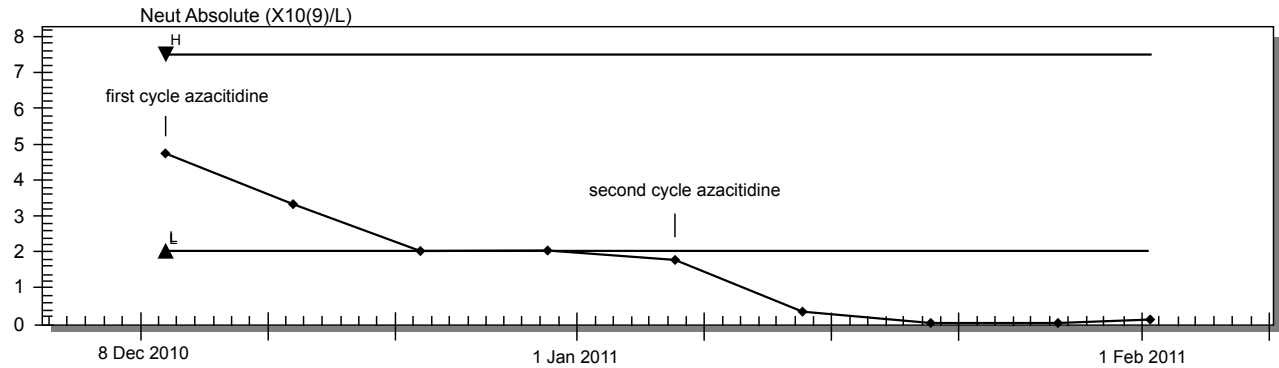

$1 \mathrm{C}$

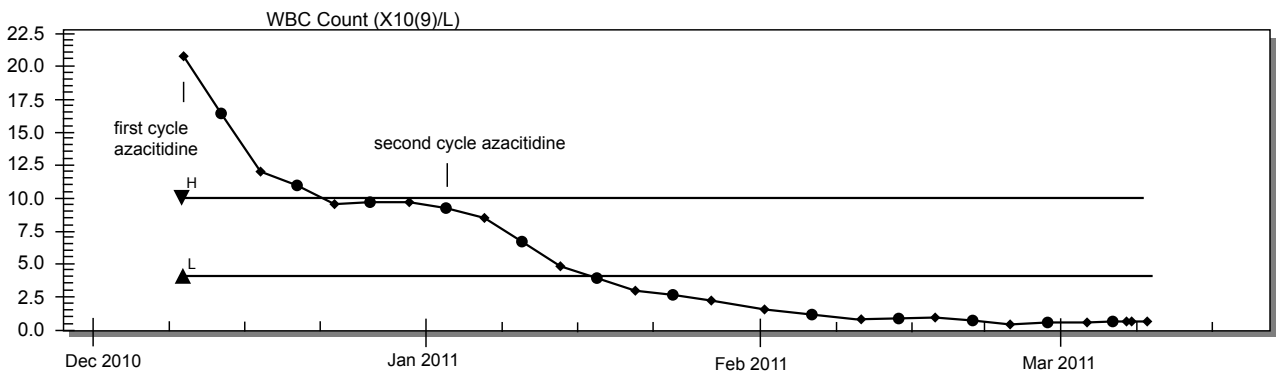

Figure 1: Prolonged leucopenia developed after second cycle of azacitidine and persisted for 9 weeks without any sign of white blood cell (WBC) recovery (1A) with persistent neutropenia (1B) and reducing blast count (1C).

transplantation [7]. This raised a concern about the effect of marrow fibrosis on hematologic recovery after intensive chemotherapy and newer cytotoxic agents such as azacitidine. In a large multicenter AZA001 trial, azacitidine has been demonstrated to prolong overall survival in patients with higher-risk MDS compared with conventional care which included best supportive care, low dose cytarabine and intensive chemotherapy [5]. This study however did not report the influence of bone marrow fibrosis on the treatment outcome and hematologic adverse events of azacitidine in MDS. In fact, to our knowledge, there is no clinical study reviewing the outcome of patients with severe marrow fibrosis treated with azacitidine. The use of azacitidine has been extended to other hematologic neoplasms including AML and MF [4,8-10]. Limited therapeutic activity of azacitidine was observed in MF with a significant percentage of patients developing grade $3 / 4$ cytopenias $[9,10]$. However the duration of cytopenia was not reported in these studies. A recent study on 54 patients with MPN transformed to MDS or AML included 7 cases of MF [4]. The study reported an overall response rate of $52 \%$ and a median overall survival of 11 months. Among the cases with MF, there was a 57\% response rate to azacitidine. However, the incidence and duration of grade $3 / 4$ cytopenias in these 7 patients with MF were not reported.

Our case illustrates severe prolonged neutropenia as an adverse effect of azacitidine in an elderly patient with MF transformed to AML which precluded him from further cycles of azacitidine. In higher-risk MDS or AML with low blast counts, clinical response with azacitidine was usually achieved after at least 4 cycles of treatment [5]. Therefore factors leading to early discontinuation of azacitidine treatment will affect the overall response and survival. A severe and prolonged treatment-related neutropenia would likely be a negative impact factor 
Citation: Yiu RC, Shan Kam GL (2012) Azacitidine Causes Prolonged Neutropenia in an Elderly Patient with Primary Myelofibrosis Transformed To Acute Myeloid Leukemia. 1: 200. doi:10.4172/scientificreports.200

on the overall outcome and treatment response and increase the risk of infective complications. Elderly patients with leukemic transformation of MF have poor survival and have very limited therapeutic options available. Most of them receive best supportive care with a median survival of less than 3 months [2]. Although azacitidine shows promising efficacy and is relatively well tolerated in MDS and AML, there are unanswered questions on its hematologic toxicity in MF. This association between the severity of marrow fibrosis and hematologic recovery after use of hypomethylating agents needs to be further studied in order to determine the overall benefit of hypomethylating agents in leukemic transformation of MF or in MDS with significant marrow fibrosis.

\section{References}

1. Dupriez B, Morel P, Demory JL, Lai JL, Simon M (1996) Prognostic factors in agnogenic myeloid metaplasia: a report on 195 cases with a new scoring system. Blood 88: 1013-1018.

2. Mesa RA, Li CY, Ketterling RP, Schroeder GS, Knudson RA (2005) Leukemic transformation in myelofibrosis with myeloid metaplasia: a single-institution experience with 91 cases. Blood 105: 973-977.

3. Appelbaum FR, Gundacker H, Head DR, Slovak ML, Willman CL (2006) Age and acute myeloid leukemia. Blood 107: 3481-3485.
4. Thepot S, Itzykson R, Seegers V, Raffoux E, Quesnel B (2010) Treatment of progression of Philadelphia-negative myeloproliferative neoplasms to myelodysplastic syndrome or acute myeloid leukemia by azacitidine: a report on 54 cases on the behalf of the Groupe Francophone des Myelodysplasies (GFM). Blood 116: 3735-3742.

5. Fenaux P, Mufti GJ, Hellstrom-Lindberg E, Santini V, Finelli C (2009) Efficacy of azacitidine compared with that of conventional care regimens in the treatment of higher-risk myelodysplastic syndromes: a randomized, open-label, phase III study. Lancet Oncol 10: 223-232.

6. Seymour JF, Fenaux P, Silverman LR, Mufti GJ, Hellstrom-Lindberg E (2010) Effects of azacitidine compared with conventional care regimens in elderly ( $\geq 75$ years) patients with higher-risk myelodysplastic syndromes. Crit Rev Oncol Hematol 76: 218-227.

7. Rajantie J, Sale GE, Deeg HJ, Amos D, Appelbaum F (1986) Adverse effects of severe marrow fibrosis on hematologic recovery after chemoradiotherapy and allogeneic bone marrow transplantation. Blood 67: 1693-1697.

8. Sudan N, Rossetti JM, Shadduck RK, Latsko J, Lech JA (2006) Treatment of acute myelogenous leukemia with outpatient azacitidine. Cancer 107: 18391843.

9. Quintas-Cardama A, Tong W, Kantarjian H, Thomas D, Ravandi F (2008) A phase II study of 5 -azacitidine for patients with primary and post-essential thrombocythemia/ polycythemia vera myelofibrosis. Leukemia 22: 965-970.

10. Mesa RA, Verstovsek S, Rivera C, Pardanani A, Hussein K (2009) 5-azacitidine has limited therapeutic activity in myelofibrosis. Leukemia 23: 180-182. 